\title{
Long-Time Simulation of a Size-Structured Population Model with a Dynamical Resource
}

\author{
L. M. Abia ${ }^{1}$, O. Angulo ${ }^{2}$, J. C. López-Marcos ${ }^{1}$ and M. A. López-Marcos ${ }^{1 *}$ \\ ${ }^{1}$ Departamento de Matemática Aplicada. Facultad de Ciencias \\ Universidad de Valladolid, Paseo Prado de la Magdalena sn., 47005. Valladolid. Spain \\ 2 Departamento de Matemática Aplicada, Escuela Universitaria Politécnica \\ Universidad de Valladolid, C/ Fco. Mendizabal 1, 47014 Valladolid, Spain
}

\begin{abstract}
In this paper, we study the numerical approximation of a size-structured population model whose dependency on the environment is managed by the evolution of a vital resource. We show that this is a difficult task: some numerical methods are not suitable for a long-time integration. We analyze the reasons for the failure.
\end{abstract}

Key words: size-structured population model, dynamical resource, numerical methods AMS subject classification: 35Q80, 92D25, 92D40, 65M25, 35B40

\section{Introduction}

Physiologically structured population models are based on the use of one or more attributes that structures the individuals in the population. Size is one of the natural and important attributes structuring the population for many species: typical examples being fish and trees. In such species the ability of an individual to obtain the necessary resources to survive and reproduce depends strongly on its size. The structured population models reflect the effect of the physiological state of the individuals on the population dynamics. In addition, the use of nonlinear structured population models allows us to take into account the effect of the competition for natural resources in the structured-specific mortality and fertility rates. We can find an extensive study of structured population dynamics models (both physiologically and age), with analytical studies of aspects such as

*Corresponding author. E-mail: malm@mac.uva.es 
existence and uniqueness, smoothness and the asymptotic behavior of solutions in $[15,11,8,7,12]$.

In this paper, we consider a size-structured population model nonlinearly coupled with an integro-ordinary differential equation accounting for substrate consumption and/or product formation as was introduced first in [13] for modeling a Daphnia magna population. The numerical solution of such a model, due to its obvious mathematical complexity, poses a serious challenge. Our starting point is a numerical method which has been successfully employed in the integration of size-structured models [5]. However, we observe that the method does not work properly for a long-time simulation. This kind of simulation has a high biological interest for these problems as we can see in $[6,4,3]$. Here, we analyze different numerical alternatives in order to circumvent the difficulties.

The paper is organized as follows: we introduce the model considered in Section 2. The test problem we use is described in Section 3. There, we also describe the equilibrium state of the test problem. In Section 4, we consider a suitable modification of the successful scheme employed for the solution of general size-structured population models. A long-time integration reveals that the numerical solution becomes unstable. We analyze the reasons for the failure and we introduce new numerical procedures in the three different (but coupled) numerical processes: the generation of the grid points, the integration of the partial differential equation and the numerical solution of the resource. Section 5 is devoted to a new numerical method which overcomes all of the difficulties. Finally, conclusions are included in Section 6.

\section{The model}

We consider the numerical study of a size-structured population model that is nonlinearly coupled with an integro-differential equation modeling the evolution of a natural resource for which individuals in the population compete. Also, we take into account a time dependent maximum size. In particular, we consider models that consist of a nonlinear partial differential equation (the population balance law)

$$
u_{t}+(g(x, S(t), t) u)_{x}=-\mu(x, S(t), t) u, \quad 0<x<x_{M}(t), \quad t>0,
$$

a nonlinear and nonlocal boundary condition that represents the birth law

$$
g(0, S(t), t) u(0, t)=\int_{0}^{x_{M}(t)} \alpha(x, S(t), t) u(x, t) d x, \quad t>0,
$$

and an initial condition

$$
u(x, 0)=u_{0}(x), \quad 0 \leq x \leq x_{M}(0),
$$

coupled with an initial value problem that represents the dynamics of the competition resource

$$
\begin{aligned}
S^{\prime}(t) & =f(S(t), I(t), t), \quad t>0, \\
S(0) & =S^{0}
\end{aligned}
$$


where the nonlocal term $I(t)$ is defined by

$$
I(t)=\int_{0}^{x_{M}(t)} \gamma(x, S(t), t) u(x, t) d x, \quad t \geq 0 .
$$

The independent variables $x$ and $t$ represent size and time, respectively. The dependent variable $u(x, t)$ is the size-specific density of individuals with size $x$ at time $t$.

As a measure of the population, with biological meaning, we consider the total population function

$$
P(t)=\int_{0}^{x_{M}(t)} u(x, t) d x, \quad t \geq 0 .
$$

We also assume that the size of any individual varies according to the following ordinary differential equation

$$
\frac{d x}{d t}=g(x, S(t), t)
$$

and thus the maximum size $x_{M}(t)$, that an individual could have at time $t$, is the solution of

$$
\left\{\begin{array}{l}
\frac{d}{d t} x_{M}(t)=g\left(x_{M}(t), S(t), t\right), \quad t>0 \\
x_{M}(0)=x_{M}
\end{array}\right.
$$

Functions $g, \alpha$ and $\mu$ represent the growth, fertility and mortality rate, respectively. These are usually called the vital functions and define the life history of an individual. Functions $\alpha$ and $\mu$ are non-negative. Note that all the vital functions $(g, \mu$ and $\alpha$ ) depend on the size $x$ (the internal structuring variable), on the value of the resource that can reflect the competition for this physiological resource or the influence of the environmental changes on the vital functions and, explicitly, on time $t$. The function $f$ on the right-hand side of (2.4) depends on the value of the resource, on the total amount of individuals in the population by means of the weighted function $I(t)$ (which represents the way of weighting the size distribution density in order to model the different influence of the individuals of different sizes on life conditions) and on time $t$.

The numerical solution of this complex model is a difficult task. There exists a technique called Escalator Boxcar Train, proposed in [13] that is the usual way in which the numerical simulation of these models has been made. Such numerical method consists of approximating the differential equations that govern the evolution of a set of moments that represents the density function. However, this technique does not provide complete information about the solution, as for example: the evolution of the size density function and the maximum size of individuals. In order to get this lost information, we consider a direct integration of the system (2.1)-(2.6) by means of the adjustment of a numerical method successfully employed in [5] for the numerical solution of sizestructured population models (in which the dynamics of the resource is not included). More details about the numerical integration of physiologically-structured population models can be found in the reviews [1,2] and the references therein, and the recent works [14, 9]. 


\section{Test problem}

In the numerical simulation, we consider the particular case of a model describing the dynamics of a population of ectothermic invertebrates. This is the case, for example, of the water flea Daphnia magna, feeding on a dynamical algal population, presented by Kooijman and Metz [10] and used in [13] as an example of the application of the Escalator Boxcar Train method. The model is described by the system (2.1)-(2.6) with

$$
\begin{aligned}
g(x, S, t) & =g\left(\frac{S}{1+S}-x\right), \\
\mu(x, S, t) & =\mu, \\
\alpha(x, S, t) & =\alpha \frac{S}{1+S} x^{2}, \\
f(S, I, t) & =r S\left(1-\frac{S}{K}\right)-I \frac{S}{1+S}, \\
\gamma(x, S, t) & =x^{2} .
\end{aligned}
$$

The model possesses the following steady state: the maximum size of the equilibrium population is

$$
x_{M}^{*}=\sqrt[3]{\frac{\mu(\mu+g)(\mu+2 g)}{2 \alpha g^{2}}},
$$

the resource at the equilibrium state is

$$
S^{*}=\frac{x_{M}^{*}}{1-x_{M}^{*}}
$$

the population density is

$$
u^{*}(x)=\frac{\alpha r}{g}\left(1+S^{*}\right)\left(1-\frac{S^{*}}{K}\right)\left(1-\frac{x}{x_{M}^{*}}\right)^{\frac{\mu}{g}-1}, \quad 0 \leq x \leq x_{M}^{*},
$$

(we assume $\mu \geq g$, in order to have a nonsingular steady state) and the weighted population

$$
I^{*}=r\left(1+S^{*}\right)\left(1-\frac{S^{*}}{K}\right) \text {. }
$$

The corresponding equilibrium state of the total population is characterized by (3.7) and

$$
P^{*}=\frac{\alpha r}{\mu} S^{*}\left(1-\frac{S^{*}}{K}\right)
$$




\section{Two-step numerical scheme based on the Midpoint Rule (MP method)}

In this section, we consider a simple modification of the scheme employed in [5] for the solution of general size-structured population models. This scheme integrates the problem along the characteristic curves. Therefore, first we rewrite the partial differential equation (2.1) in a more suitable form for its numerical treatment. So we define

$$
\mu^{*}(x, z, t)=\mu(x, z, t)+g_{x}(x, z, t)
$$

thus equation (2.1) has the form

$$
u_{t}(x, t)+g(x, S(t), t) u_{x}(x, t)=-\mu^{*}(x, S(t), t) u(x, t), \quad 0<x<x_{M}(t), \quad t>0 .
$$

We denote by $x\left(t ; t^{*}, x^{*}\right)$ the characteristic curve of equation (4.1) which takes the value $x^{*}$ at time $t^{*}$. Such a characteristic curve is the solution of the initial value problem

$$
\left\{\begin{array}{l}
\frac{d}{d t} x\left(t ; t^{*}, x^{*}\right)=g\left(x\left(t ; t^{*}, x^{*}\right), S(t), t\right), \quad t \geq t^{*} \\
x\left(t^{*} ; t^{*}, x^{*}\right)=x^{*}
\end{array}\right.
$$

Now, we consider the function that represents the solution of (4.2) along that characteristic curve

$$
w\left(t ; t^{*}, x^{*}\right)=u\left(x\left(t ; t^{*}, x^{*}\right), t\right), \quad t \geq t^{*},
$$

that satisfies the initial value problem

$$
\left\{\begin{aligned}
\frac{d}{d t} w\left(t ; t^{*}, x^{*}\right) & =-\mu^{*}\left(x\left(t ; t^{*}, x^{*}\right), S(t), t\right) w\left(t ; t^{*}, x^{*}\right), \quad t \geq t^{*}, \\
w\left(t^{*} ; t^{*}, x^{*}\right) & =u\left(x^{*}, t^{*}\right),
\end{aligned}\right.
$$

and, therefore, it can be represented in the following integral form

$$
w\left(t ; t^{*}, x^{*}\right)=u\left(x^{*}, t^{*}\right) \exp \left\{-\int_{t^{*}}^{t} \mu^{*}\left(x\left(\tau ; t^{*}, x^{*}\right), S(\tau), \tau\right) d \tau\right\}, \quad t \geq t^{*} .
$$

We carry out the time integration by using a constant step $k>0$. Then, we introduce the discrete time levels $t^{n}=n k, n=0,1,2, \ldots$ The parameter of the discretization related to the size variable is $J$, a positive integer describing the number of grid points. Thus, the numerical method is a recursive procedure that provides, at each discrete time level, a mesh grid on the size interval, the approximation to the density on such a mesh grid (from the known approximations at previous discrete time levels) and the approximation to the resource. More precisely, for each time level $n=0,1,2, \ldots$, the numerical method produces a grid of points $\mathbf{X}^{n}$ on the size interval, a vector $\mathbf{U}^{n}$ with the approximations to the solution of the problem at the grid points and time $t^{n}$, and $S^{n}$ the numerical value of the resource. 
We start the procedure at time $t^{0}=0$, by taking a uniform mesh grid on the interval $\left[0, x_{M}\right]$. The diameter of such a mesh grid is $h=x_{M} / J$, and the initial grid nodes are $X_{j}^{0}=j h, 0 \leq j \leq J$. We consider as an approximation to the density at this time level, the grid restriction of the initial condition in (2.3), that is, $U_{j}^{0}=u_{0}\left(X_{j}^{0}\right), 0 \leq j \leq J$. Finally, we use $S^{0}$ in (2.5) as the initial value of the resource.

Discretization of equations (4.2), (4.3), (2.2), (2.4), (2.6) provides the recursive procedure. To this end, in this section we consider a discretization based on the midpoint rule that provides a two-step method, that is, the numerical solution at time $t^{n+2}$ is obtained from the known values of the numerical solution at the time values $t^{n+1}$ and $t^{n}$. Taking into account that initially we only know the approximation at time $t^{0}=0$, the first step of the method must be carried out by using a one-step procedure. To this end, we use a one-step method based on the Modified Euler scheme.

As we will see, in each step the dimension of the numerical vectors that provide the approximations at the new time level is increased with respect to those at the previous level. So, in order to avoid an unbroken increase in the dimension and the corresponding computational effort, after the step we reduce the dimension of the computed vectors by means of a grid selection.

In the description of the method, we use the following notation: vectors $\boldsymbol{\alpha}^{p}$ and $\gamma^{p}$ contain the evaluations of the functions $\alpha$ and $\gamma$ in (2.2) and (2.6), respectively, at the grid points in $\mathbf{X}^{p}$, at the resource value $S^{p}$ and at time $t^{p}$. Thus, these vectors possess the same dimension as the corresponding $\mathbf{X}^{p}$. Products $\boldsymbol{\gamma}^{p}$. $\mathbf{U}^{p}$ and $\boldsymbol{\alpha}^{p}$. $\mathbf{U}^{p}$ must be considered componentwise. In order to approximate integrals over the interval $\left[0, x_{M}\left(t^{p}\right)\right]$, we use the composite trapezoidal quadrature rule based on the grid points $\mathbf{X}^{p}=\left[X_{0}^{p}, X_{1}^{p}, \ldots, X_{M}^{p}\right]$, that is

$$
\mathcal{Q}\left(\mathbf{X}^{p}, \mathbf{V}^{p}\right)=\sum_{j=1}^{M} \frac{X_{j}^{p}-X_{j-1}^{p}}{2}\left(V_{j-1}^{p}+V_{j}^{p}\right) .
$$

The overall method can be written

\section{Initial condition}

Time: $\quad t^{0}=0$

Grid: $\quad \mathbf{X}^{0}=\left[X_{0}^{0}, X_{1}^{0}, \ldots, X_{J}^{0}\right]$

$$
X_{j}^{0}=j h, \quad 0 \leq j \leq J
$$

Resource: $\quad S^{0}$

Density: $\quad \mathbf{U}^{0}=\left[U_{0}^{0}, U_{1}^{0}, \ldots, U_{J}^{0}\right]$

$$
U_{j}^{0}=u_{0}\left(X_{j}^{0}\right), \quad 0 \leq j \leq J .
$$




\section{First step}

\section{Stage 1}

Time: $\quad t^{1 / 2}=\frac{k}{2}$

Grid: $\quad \mathbf{X}^{1 / 2}=\left[X_{0}^{1 / 2}, X_{1}^{1 / 2}, \ldots, X_{J+1}^{1 / 2}\right]$

$X_{0}^{1 / 2}=0$

$X_{j+1}^{1 / 2}=X_{j}^{0}+\frac{k}{2} g\left(X_{j}^{0}, S^{0}, t^{0}\right), \quad 0 \leq j \leq J$

Resource: $\quad S^{1 / 2}=S^{0}+\frac{k}{2} f\left(S^{0}, \mathcal{Q}\left(\mathbf{X}^{0}, \gamma^{0} \cdot \mathbf{U}^{0}\right), t^{0}\right)$

Density: $\quad \mathbf{U}^{1 / 2}=\left[U_{0}^{1 / 2}, U_{1}^{1 / 2}, \ldots, U_{J+1}^{1 / 2}\right]$

$$
\begin{aligned}
U_{0}^{1 / 2} & =\frac{\mathcal{Q}\left(\mathbf{X}^{1 / 2}, \boldsymbol{\alpha}^{1 / 2} \cdot \mathbf{U}^{1 / 2}\right)}{g\left(X_{0}^{1 / 2}, S^{1 / 2}, t^{1 / 2}\right)} \\
U_{j+1}^{1 / 2} & =U_{j}^{0} \exp \left\{-\frac{k}{2} \mu^{*}\left(X_{j}^{0}, S^{0}, t^{0}\right)\right\}, \quad 0 \leq j \leq J
\end{aligned}
$$

\section{Stage 2}

Time: $\quad t^{1}=k$

Grid: $\quad \mathbf{X}^{1}=\left[X_{0}^{1}, X_{1}^{1}, \ldots, X_{J+1}^{1}\right]$

$X_{0}^{1}=0$

$X_{j+1}^{1}=X_{j}^{0}+k g\left(X_{j+1}^{1 / 2}, S^{1 / 2}, t^{1 / 2}\right), \quad 0 \leq j \leq J$

Resource: $\quad S^{1} \quad=\quad S^{0}+k f\left(S^{1 / 2}, \mathcal{Q}\left(\mathbf{X}^{1 / 2}, \boldsymbol{\gamma}^{1 / 2} \cdot \mathbf{U}^{1 / 2}\right), t^{1 / 2}\right)$

Density: $\quad \mathbf{U}^{1}=\left[U_{0}^{1}, U_{1}^{1}, \ldots, U_{J+1}^{1}\right]$

$$
\begin{aligned}
U_{0}^{1} & =\frac{\mathcal{Q}\left(\mathbf{X}^{1}, \boldsymbol{\alpha}^{1} \cdot \mathbf{U}^{1}\right)}{g\left(X_{0}^{1}, S^{1}, t^{1}\right)} \\
U_{j+1}^{1} & =U_{j}^{0} \exp \left\{-k \mu^{*}\left(X_{j+1}^{1 / 2}, S^{1 / 2}, t^{1 / 2}\right)\right\}, \quad 0 \leq j \leq J
\end{aligned}
$$


General step $(n \geq 0)$

$$
\begin{aligned}
& \begin{array}{l}
\begin{array}{l}
\text { Intermediate } \\
\text { values: }
\end{array} t^{n+3 / 2}=t^{n+1}+\frac{k}{2} \\
\text { nat }
\end{array} \\
& X_{1}^{n+3 / 2}=X_{0}^{n+1}+\frac{k}{2} g\left(X_{0}^{n+1}, S^{n+1}, t^{n+1}\right) \\
& S^{n+3 / 2}=S^{n+1}+\frac{k}{2} f\left(S^{n+1}, \mathcal{Q}\left(\mathbf{X}^{n+1}, \gamma^{n+1} \cdot \mathbf{U}^{n+1}\right), t^{n+1}\right) \\
& \text { Time: } \quad t^{n+2}=t^{n+1}+k \\
& \text { Grid: } \quad \mathbf{X}^{n+2}=\left[X_{0}^{n+2}, X_{1}^{n+2}, \ldots, X_{J+1}^{n+2}, X_{J+2}^{n+2}\right] \\
& X_{0}^{n+2}=0 \\
& X_{1}^{n+2}=X_{0}^{n+1}+k g\left(X_{1}^{n+3 / 2}, S^{n+3 / 2}, t^{n+3 / 2}\right), \\
& X_{j+2}^{n+2}=X_{j}^{n}+2 k g\left(X_{j+1}^{n+1}, S^{n+1}, t^{n+1}\right), \quad 0 \leq j \leq J \\
& \text { Resource: } \quad S^{n+2}=S^{n}+2 k f\left(S^{n+1}, \mathcal{Q}\left(\mathbf{X}^{n+1}, \gamma^{n+1} \cdot \mathbf{U}^{n+1}\right), t^{n+1}\right) \\
& \text { Density: } \quad \mathbf{U}^{n+2}=\left[U_{0}^{n+2}, U_{1}^{n+2}, \ldots, U_{J+1}^{n+2}, U_{J+2}^{n+2}\right] \\
& U_{0}^{n+2}=\frac{\mathcal{Q}\left(\mathbf{X}^{n+2}, \boldsymbol{\alpha}^{n+2} \cdot \mathbf{U}^{n+2}\right)}{g\left(X_{0}^{n+2}, S^{n+2}, t^{n+2}\right)} \\
& U_{1}^{n+2}=U_{0}^{n+1} \exp \left\{-k \mu^{*}\left(X_{1}^{n+3 / 2}, S^{n+3 / 2}, t^{n+3 / 2}\right)\right\}, \\
& U_{j+2}^{n+2}=U_{j}^{n} \exp \left\{-2 k \mu^{*}\left(X_{j+1}^{n+1}, S^{n+1}, t^{n+1}\right)\right\}, 0 \leq j \leq J .
\end{aligned}
$$

Note that the general step of the method increases the number of grid points and also the dimension of the vector with the numerical densities: at time $t^{n}$ we have $J$ grid nodes in $\mathbf{X}^{n}$ and the $J$-dimensional vector $\mathbf{U}^{n}$, at time $t^{n+1}$ we have $J+1$ grid nodes in $\mathbf{X}^{n+1}$ and the $(J+1)$ dimensional vector $\mathbf{U}^{n+1}$, and at time $t_{n+2}$ we obtain $J+2$ grid nodes in $\mathbf{X}^{n+2}$ and the $(J+2)$ dimensional vector $\mathbf{U}^{n+2}$. In order to maintain the number of grid points suitable to perform the next step, we eliminate at time $t^{n+2}$ the first grid node $X_{l}^{n+2}$ that satisfies

$$
\left|X_{l+1}^{n+2}-X_{l-1}^{n+2}\right|=\min _{1 \leq j \leq J+1}\left|X_{j+1}^{n+2}-X_{j-1}^{n+2}\right|
$$

and we also remove the grid node over the same characteristic curve at the previous time $t^{n+1}$, that is $X_{l-1}^{n+1}$. We perform the same reduction in the corresponding vectors $\mathbf{U}^{n+2}$ and $\mathbf{U}^{n+1}$.

We also note that the method seems to be implicit: all the expressions provide explicit equations for the numerical values at the highest time level, except those which involve the numerical density $U_{0}^{p}$ at the first grid point. However, we can implement the method in explicit form. If we first compute the values of the numerical density $U_{j}^{p}$ at grid points $j \geq 1$, and we take into account that 
the quadrature rule $\mathcal{Q}\left(\mathbf{X}^{p}, \boldsymbol{\alpha}^{p} . \mathbf{U}^{p}\right)$ produces a linear combination of the density values, then we can derive an explicit expression for $U_{0}^{p}$ based on known values of the approximation.

In order to test the numerical method, we consider the simulation of the problem described in Section 3: system (2.1)-(2.6) with the functions (3.1)-(3.5). The parameters are taken in order to ensure that the equilibrium solution described by $x_{M}^{*}, S^{*}$ and $u^{*}(x)$ and characterized by (3.6)(3.8) is asymptotically stable. Moreover, we assume that the equilibrium density (3.8) is bounded and vanishes at the maximum individual size. So, as in the experiments of [13], we take $\mu=$ $0.1, \alpha=0.75$. The equilibrium values of the maximum individual size and the resource are $x_{M}^{*} \approx 0.80338075$ and $S^{*} \approx 4.08597212$. In order to ensure that the equilibrium density (3.8) is nonsingular and vanishes at the maximum individual size, we assume that $g<\mu=0.1$. On the other hand, taking into account the values chosen for $\alpha$ and $\mu$, the biological restriction $S^{*}>0$, that is equivalent to $x_{M}^{*}<1$, requires that $g>\frac{3 \mu^{2}+\sqrt{\mu^{3}(\mu-8 \alpha)}}{4(\alpha-\mu)} \approx 0.0416$. So, we choose $g=0.075$ that satisfies both conditions. As in [13], we take $r=3$ and $K=8.3$. In our case, this value of $K$ makes the equilibrium asymptotically stable. The total population of the equilibrium state is $P^{*} \approx 46.67638671$.

The initial maximum individual size chosen is $x_{M}=1$, and the initial conditions in (2.5) and (2.3), small perturbations of (3.7) and (3.8), respectively. To this end, we carry out the following steps

- Choose $\widetilde{x}_{M}<x_{M}$ close to the equilibrium value $x_{M}^{*}$ in (3.6).

- Compute the initial value of the resource $S^{0}=\frac{\widetilde{x}_{M}}{1-\widetilde{x}_{M}}$, perturbed counterpart of (3.7).

- Consider, in a similar way that (3.8), the initial density function as

$$
u_{0}(x)=\left\{\begin{array}{cl}
\frac{\alpha r}{g}\left(1+S^{0}\right)\left(1-\frac{S^{0}}{K}\right)\left(1-\frac{x}{\widetilde{x}_{M}}\right)^{\beta} & \text { if } 0 \leq x \leq \widetilde{x}_{M}, \\
0 & \text { if } \widetilde{x}_{M}<x \leq x_{M} .
\end{array}\right.
$$

The value of $\beta$ is taken in order to ensure compatibility between the initial condition $u_{0}$ in (2.3) and the boundary condition (2.2). In this case, this condition reads

$$
(\beta+3)(\beta+2)(\beta+1)=\frac{2 \alpha \widetilde{x}_{M}^{3}}{g} .
$$

As a first simulation, we choose $\widetilde{x}_{M}=0.8$, that provides the value $S^{0}=4$. Note that this initial value of the resource is less than the value at the equilibrium, but very close $\left(\left|S^{0}-S^{*}\right| \approx\right.$ $8.6 \times 10^{-2}$ ). The exponent required in (4.6) is $\beta \approx 0.32479770$ and, then, the initial total population is $P(0) \approx 46.92691934$ (the initial total population is greater than the values at the equilibrium but close $\left.\left|P(0)-P^{*}\right| \approx 2.5 \times 10^{-1}\right)$.

The parameters of the discretization for the MP method are $h=2 \times 10^{-4}(J=5000)$ and $k=10^{-4}$. In Figure 1, we present the numerical approximation obtained with the MP method. 

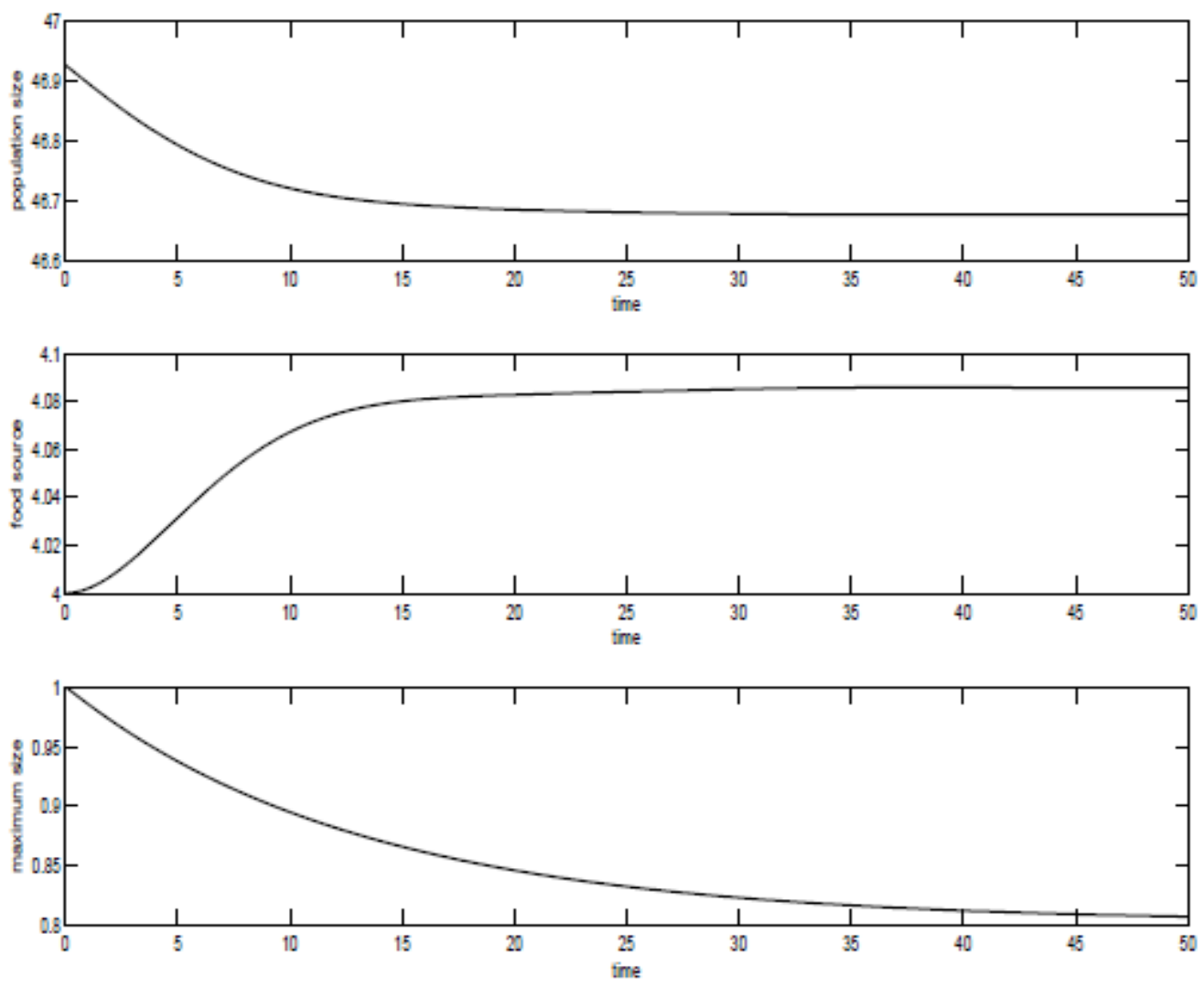

Figure 1: Evolution of the numerical solution obtained with the MP method. Short-time integration.

The first picture shows the evolution along the time of the total population computed from the numerical density by using the composite trapezoidal quadrature rule. The second picture refers to the evolution of the dynamical resource, and the third corresponds to the evolution of the maximum individual size. We can observe that, in this time interval, the numerical solution evolves towards the equilibrium state. However, if we carry out a longer time integration, we observe that the numerical solution becomes unstable. This behavior can observed in Figure 2.

But this is not the real situation. The use of a non A-stable method for the numerical simulation of an asymptotically stable steady state of the differential equation (2.4) that governs the dynamics of the resource may be the reason for the failure. We are approximating the steady state of the equation

$$
y^{\prime}=\phi(y)
$$

Such a steady state $y^{*}$ is asymptotically stable, so $\lambda=\phi_{y}\left(y^{*}\right)<0$. When we use the midpoint rule

$$
y^{n+2}=y^{n}+2 k \phi\left(y^{n+1}\right),
$$



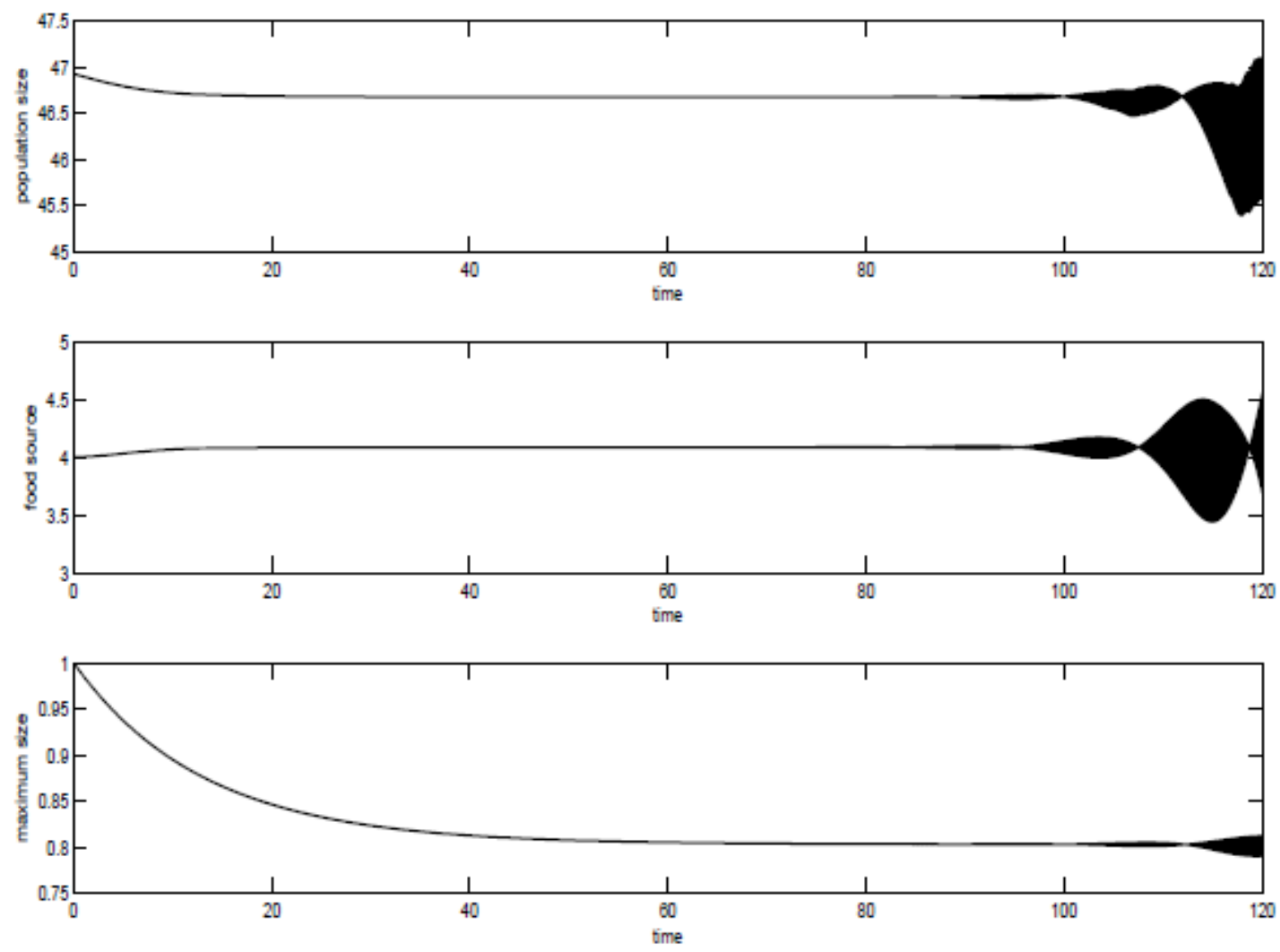

Figure 2: Evolution of the numerical solution obtained with the MP method. Long time integration.

the difference between the computed solution and the steady state $e^{n}=y^{n}-y^{*}$ satisfies

$$
\begin{aligned}
e^{n+2} & =e^{n}+2 k\left(\phi\left(y^{n+1}\right)-\phi\left(y^{*}\right)\right) \\
& =e^{n}+2 k \lambda e^{n+1}+O\left(\left(e^{n+1}\right)^{2}\right) .
\end{aligned}
$$

Elimination of the highest order terms provide the following linear difference equation

$$
e^{n+2}-2 k \lambda e^{n+1}-e^{n}=0 .
$$

It would be desirable that $\lim _{n \rightarrow \infty} e^{n}=0$ in order to ensure that the numerical solution mimics the behavior of the theoretical one. The solution of the difference equation depends on the roots of its characteristic equation: $r_{1}$ and $r_{2}$. In this way, if $\max \left\{\left|r_{1}\right|,\left|r_{2}\right|\right\}<1$ then: $\lim _{n \rightarrow \infty} e^{n}=0$. However, the roots are $k \lambda \pm \sqrt{(k \lambda)^{2}-1}$ and $\max \left\{\left|r_{1}\right|,\left|r_{2}\right|\right\}=\sqrt{(k \lambda)^{2}-1}-k \lambda>1$. The right behavior observed in Figure 1, is due to the convergence of the numerical solution to the theoretical one in a fixed time interval $[0, T]$ by making the parameters of the discretization sufficiently small.

Thus, the MP method is not suitable for a long-time simulation, and we try to design a new numerical method with a better behavior. As a first option, we could consider the Modified Euler method in order to integrate (2.4). 
If we analyze the A-stability of the Modified Euler method

$$
\begin{aligned}
y^{n+1 / 2} & =y^{n}+\frac{k}{2} \phi\left(y^{n}\right), \\
y^{n+1} & =y^{n}+k \phi\left(y^{n+1 / 2}\right),
\end{aligned}
$$

for the differential equation (4.7), the difference between the computed solution and the steady state $e^{n}=y^{n}-y^{*}$ satisfies

$$
\begin{aligned}
e^{n+1} & =e^{n}+k\left(\phi\left(y^{n}+\frac{k}{2} \phi\left(y^{n}\right)\right)-\phi\left(y^{*}+\frac{k}{2} \phi\left(y^{*}\right)\right)\right) \\
& =\left(1+k \lambda+\frac{(k \lambda)^{2}}{2}\right) e^{n}+O\left(\left(e^{n}\right)^{2}\right) .
\end{aligned}
$$

Elimination of the highest order terms provides

$$
e^{n+1}=\left(1+k \lambda+\frac{(k \lambda)^{2}}{2}\right) e^{n} .
$$

In order to obtain $\lim _{n \rightarrow \infty} e^{n}=0$, it is sufficient that $k \lambda \in(-2,0)$. So, a sufficiently small value of $k$ guarantees the stability of the numerical solution.

Thus, we are going to substitute the numerical integrator to approximate the solution of (2.4) in the MP method. Now, to obtain $S^{n}$, we use the Modified Euler method. The computation of the grid and the solution is performed using the MP method obtaining the following equations for the general step (from now on, we only show the differences in each new formulation in comparison to the previous one):

\section{General step $(n \geq 0)$}

\section{Stage 1}

Time: $\quad t^{n+3 / 2}=t^{n+1}+\frac{k}{2}$

Grid: $\quad \mathbf{X}^{n+3 / 2}=\left[X_{0}^{n+3 / 2}, X_{1}^{n+3 / 2}, \ldots, X_{J+1}^{n+3 / 2}\right]$

$$
\begin{aligned}
& X_{0}^{n+3 / 2}=0 \\
& X_{j+1}^{n+3 / 2}=X_{j}^{n+1}+\frac{k}{2} g\left(X_{j}^{n+1}, S^{n+1}, t^{n+1}\right), \quad 0 \leq j \leq J
\end{aligned}
$$

Resource: $\quad S^{n+3 / 2}=S^{n+1}+\frac{k}{2} f\left(S^{n+1}, \mathcal{Q}\left(\mathbf{X}^{n+1}, \gamma^{n+1} \cdot \mathbf{U}^{n+1}\right), t^{n+1}\right)$

Density: $\quad \mathbf{U}^{n+3 / 2}=\left[U_{0}^{n+3 / 2}, U_{1}^{n+3 / 2}, \ldots, U_{J+1}^{n+3 / 2}\right]$

$$
\begin{aligned}
& U_{0}^{n+3 / 2}=\frac{\mathcal{Q}\left(\mathbf{X}^{n+3 / 2}, \boldsymbol{\alpha}^{n+3 / 2} \cdot \mathbf{U}^{n+3 / 2}\right)}{g\left(X_{0}^{n+3 / 2}, S^{n+3 / 2}, t^{n+3 / 2}\right)} \\
& U_{j+1}^{n+3 / 2}=U_{j}^{n+1} \exp \left\{-\frac{k}{2} \mu^{*}\left(X_{j}^{n+1}, S^{n+1}, t^{n+1}\right)\right\}, \quad 0 \leq j \leq J
\end{aligned}
$$




\section{Stage 2}

Time: $\quad t^{n+2}=t^{n+1}+k$

Resource: $\quad S^{n+2}=S^{n+1}+k f\left(S^{n+3 / 2}, \mathcal{Q}\left(\mathbf{X}^{n+3 / 2}, \gamma^{n+3 / 2} \cdot \mathbf{U}^{n+3 / 2}\right), t^{n+3 / 2}\right)$

The remarks about the grid selection and the explicit implementation included in the description of the MP method apply to this method.

In Figure 3, we can observe the simulation obtained with this method with the same parameters of the previous simulation $h=2 \times 10^{-4}(J=5000)$ and $k=10^{-4}$. We can observe that the method is able to make a longer time integration than the MP method but the numerical approximation still becomes unstable over time.
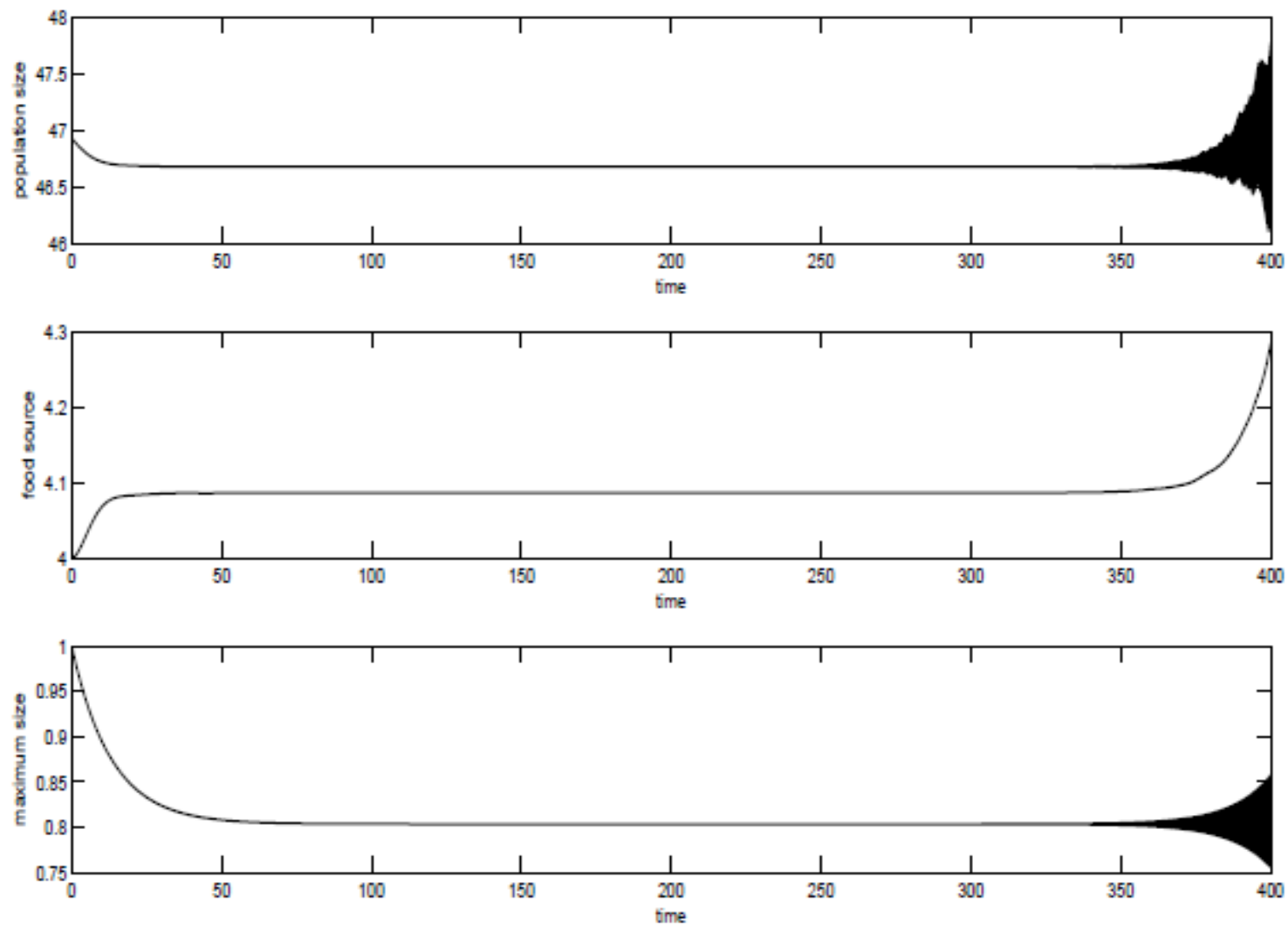

Figure 3: Evolution of the numerical solution obtained with the resource modification of the MP method. Long-time integration. 
We propose next a second modification of the MP method by changing the way in which the grid $\mathbf{X}^{n}$ is computed. In this second scheme, in order to describe the general step, the first stage is calculated as in the previous method, we keep the computation of the resource and the approximation to the solution with the equations of the MP method, and we obtain $\mathbf{X}^{n}$ by means of the stable method, resulting in

$$
\begin{aligned}
& \text { General step }(n \geq 0) \\
& X_{0}^{n+2}=0 \\
& X_{j+1}^{n+2}=X_{j}^{n+1}+k g\left(X_{j+1}^{n+3 / 2}, S^{n+3 / 2}, t^{n+3 / 2}\right), \quad 0 \leq j \leq J
\end{aligned}
$$

However, we observe an unstable behavior as with the use of the MP method. Finally, if we introduce a modification of the MP method by using a one-step method only in the computation of $\mathbf{U}^{n}$, the behavior of the solution is also unstable.

Therefore, the change in the numerical integration of the resource improves the overall numerical integration, but it does not provide a stable algorithm. This could be due to the fact that, when we perform a long-time integration, the equations in (4.2) are close to an equilibrium steady state and their numerical approximation suffers the same difficulties as the numerical integration of (2.4). Then, we introduce a new method so that, in the general step, both the grid $\mathbf{X}^{n}$ and the resource $S^{n}$ are computed with the stable method and the computation of the solution is not changed with respect to the MP method.

In Figure 4, we can observe the simulation obtained with this method with the same parameters of the previous simulations $h=2 \times 10^{-4}(J=5000)$ and $k=10^{-4}$. We observe a stable behavior for a long-time integration.

We have considered other modifications of the MP method but the resulting approximations were no better than the last one.

\section{One-step numerical scheme based on the Modified Euler Method (ME method)}

The numerical simulation carried out in the previous section encourages the use of numerical methods with better stability properties. In this way, such an experience inspires the following new method based on the Modified Euler scheme. The resulting method is a one-step method: the numerical solution at time $t^{n+1}$ is obtained from the known values of the numerical solution at time $t^{n}$. A single step of this method was used in the MP method to obtain the approximation at time $t^{1}$. 

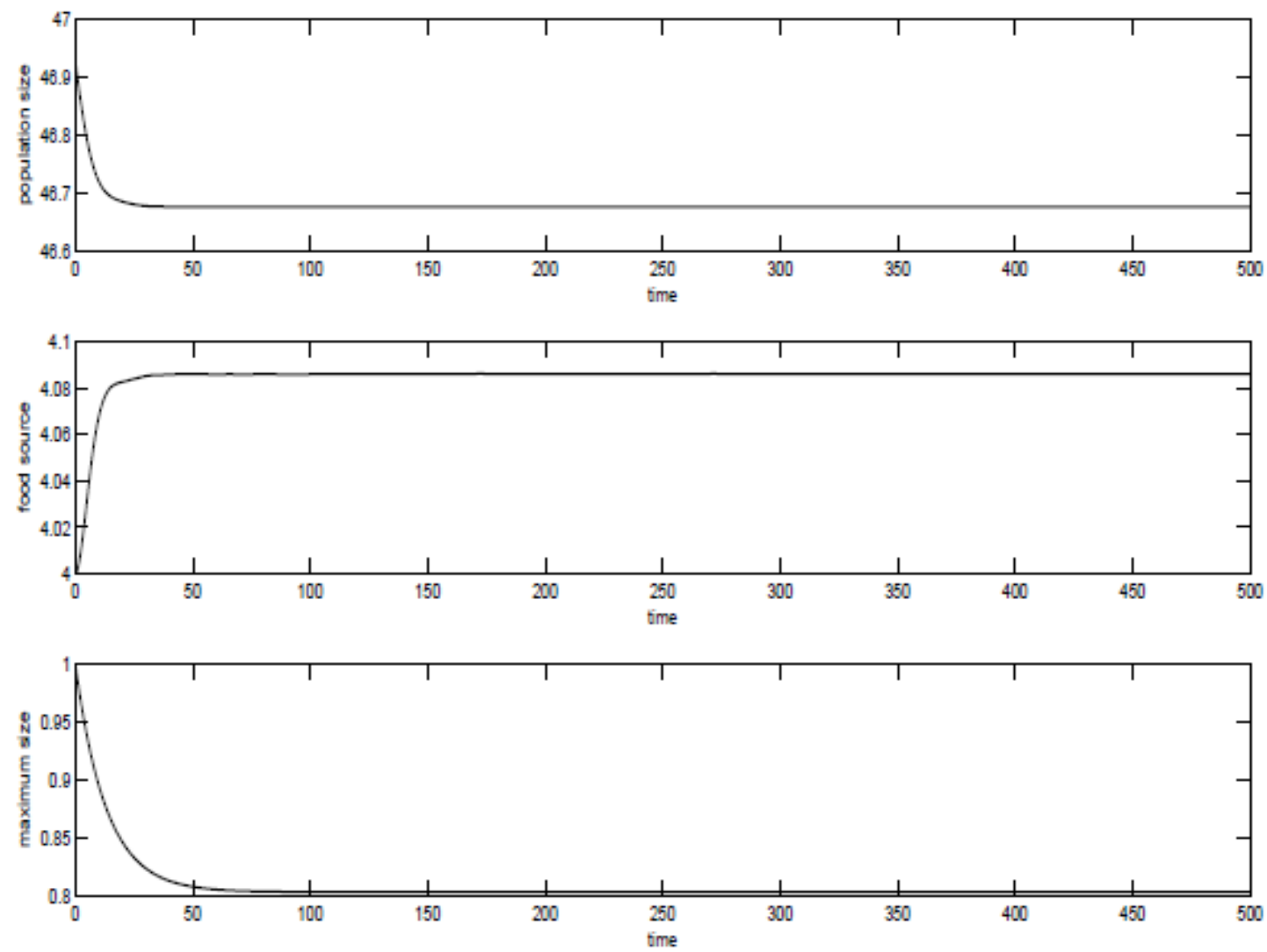

Figure 4: Evolution of the numerical solution obtained with the grid and resource modification of the MP method. Long-time integration.

By using the same notation introduced in the previous section, the overall method can be written

\section{Initial condition}

Time: $\quad t^{0}=0$

Grid: $\quad \mathbf{X}^{0}=\left[X_{0}^{0}, X_{1}^{0}, \ldots, X_{J}^{0}\right]$

$$
X_{j}^{0}=j h, \quad 0 \leq j \leq J
$$

Resource: $\quad S^{0}$

$$
\text { Density: } \quad \begin{aligned}
\mathbf{U}^{0} & =\left[U_{0}^{0}, U_{1}^{0}, \ldots, U_{J}^{0}\right] \\
U_{j}^{0} & =u_{0}\left(X_{j}^{0}\right), \quad 0 \leq j \leq J .
\end{aligned}
$$




\section{General step $(n \geq 0)$}

\section{Stage 1}

Time: $\quad t^{n+1 / 2}=t^{n}+\frac{k}{2}$

Grid: $\quad \mathbf{X}^{n+1 / 2}=\left[X_{0}^{n+1 / 2}, X_{1}^{n+1 / 2}, \ldots, X_{J+1}^{n+1 / 2}\right]$

$X_{0}^{n+1 / 2}=0$

$X_{j+1}^{n+1 / 2}=X_{j}^{n}+\frac{k}{2} g\left(X_{j}^{n}, S^{n}, t^{n}\right), \quad 0 \leq j \leq J$

Resource: $\quad S^{n+1 / 2}=S^{n}+\frac{k}{2} f\left(S^{n}, \mathcal{Q}\left(\mathbf{X}^{n}, \boldsymbol{\gamma}^{n} \cdot \mathbf{U}^{n}\right), t^{n}\right)$

Density: $\quad \mathbf{U}^{n+1 / 2}=\left[U_{0}^{n+1 / 2}, U_{1}^{n+1 / 2}, \ldots, U_{J+1}^{n+1 / 2}\right]$

$$
\begin{aligned}
U_{0}^{n+1 / 2} & =\frac{\mathcal{Q}\left(\mathbf{X}^{n+1 / 2}, \boldsymbol{\alpha}^{n+1 / 2} \cdot \mathbf{U}^{n+1 / 2}\right)}{g\left(X_{0}^{n+1 / 2}, S^{n+1 / 2}, t^{n+1 / 2}\right)} \\
U_{j+1}^{n+1 / 2} & =U_{j}^{n} \exp \left\{-\frac{k}{2} \mu^{*}\left(X_{j}^{n}, S^{n}, t^{n}\right)\right\}, \quad 0 \leq j \leq J
\end{aligned}
$$

\section{Stage 2}

Time: $\quad t^{n+1}=t^{n}+k$

Grid: $\quad \mathbf{X}^{n+1}=\left[X_{0}^{n+1}, X_{1}^{n+1}, \ldots, X_{J+1}^{n+1}\right]$

$X_{0}^{n+1}=0$

$X_{j+1}^{n+1}=X_{j}^{n}+k g\left(X_{j+1}^{n+1 / 2}, S^{n+1 / 2}, t^{n+1 / 2}\right), \quad 0 \leq j \leq J$

Resource: $\quad S^{n+1}=S^{n}+k f\left(S^{n+1 / 2}, \mathcal{Q}\left(\mathbf{X}^{n+1 / 2}, \gamma^{n+1 / 2} \cdot \mathbf{U}^{n+1 / 2}\right), t^{n+1 / 2}\right)$

Density: $\quad \mathbf{U}^{n+1}=\left[U_{0}^{n+1}, U_{1}^{n+1}, \ldots, U_{J+1}^{n+1}\right]$

$$
U_{0}^{n+1}=\frac{\mathcal{Q}\left(\mathbf{X}^{n+1}, \boldsymbol{\alpha}^{n+1} \cdot \mathbf{U}^{n+1}\right)}{g\left(X_{0}^{n+1}, S^{n+1}, t^{n+1}\right)}
$$

$U_{j+1}^{n+1}=U_{j}^{n} \exp \left\{-k \mu^{*}\left(X_{j+1}^{n+1 / 2}, S^{n+1 / 2}, t^{n+1 / 2}\right)\right\}, \quad 0 \leq j \leq J$ 
Like the MP method, the general step of the ME method increases the number of grid points and also the dimension of the vector with the numerical densities: at time $t^{n}$ we have $J$ grid nodes in $\mathbf{X}^{n}$ and the $J$-dimensional vector $\mathbf{U}^{n}$, and at time $t^{n+1}$ we obtain $J+1$ grid nodes in $\mathbf{X}^{n+1}$ and the $(J+1)$-dimensional vector $\mathbf{U}^{n+1}$. In order to maintain the number of grid points suitable to perform the next step, we eliminate at time $t^{n+1}$ the first grid node $X_{l}^{n+1}$ that satisfies

$$
\left|X_{l+1}^{n+1}-X_{l-1}^{n+1}\right|=\min _{1 \leq j \leq J+1}\left|X_{j+1}^{n+1}-X_{j-1}^{n+1}\right|
$$

We reproduce the same reduction in the corresponding vector $\mathbf{U}^{n+1}$. Again, the method seems to be implicit, but we can implement it in explicit form by following the same indications described in the previous section for the MP method.

In Figure 5, we can observe the simulation obtained with the ME method with parameters $h=2 \times 10^{-4}(J=5000)$ and $k=5 \times 10^{-4}$ (greater than the time step used in the simulation carried out with the methods described in the previous sections). We can observe the stable behavior of the solution for a long-time integration.
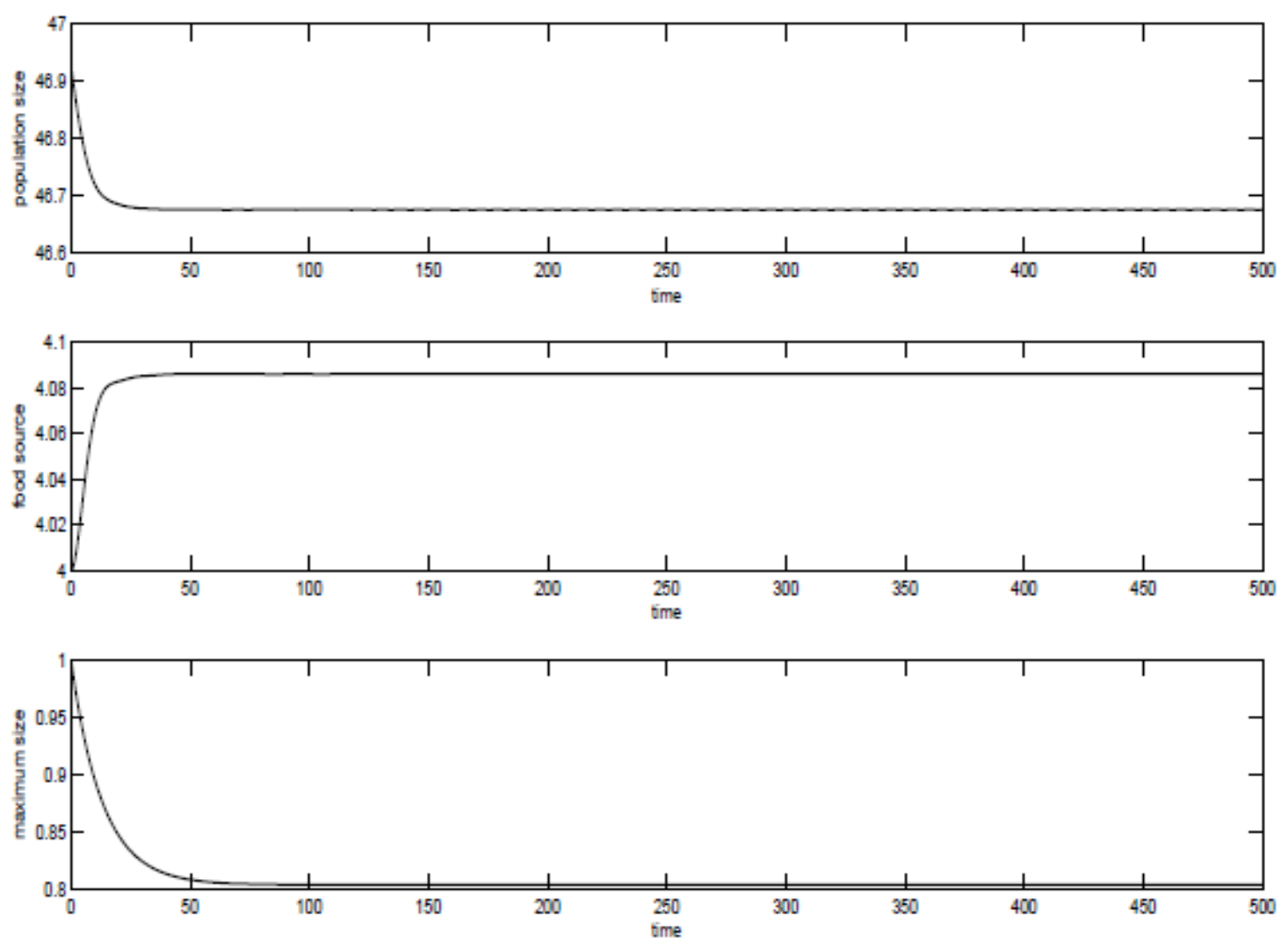

Figure 5: Evolution of the numerical solution obtained with the ME method. Stable equilibrium case, small perturbations. 
The ME method still works well in the case of greater perturbations of the steady state equilibrium. For example, by taking $\widetilde{x}_{M}=0.875$, we obtain the value $S^{0}=7$. In this case, the initial value of the resource is greater than the value at the equilibrium and $\left|S^{0}-S^{*}\right| \approx 2.9$. The exponent required in (4.6) is $\beta \approx 0.51530574$ and, then, the initial total population is $P(0) \approx 21.70622426$ (the initial total population is less than the values at the equilibrium and $\left|P(0)-P^{*}\right| \approx 25.0$ ).

Taking the values of the parameters of the discretization as in the previous experiment, we present the results obtained in Figure 6.
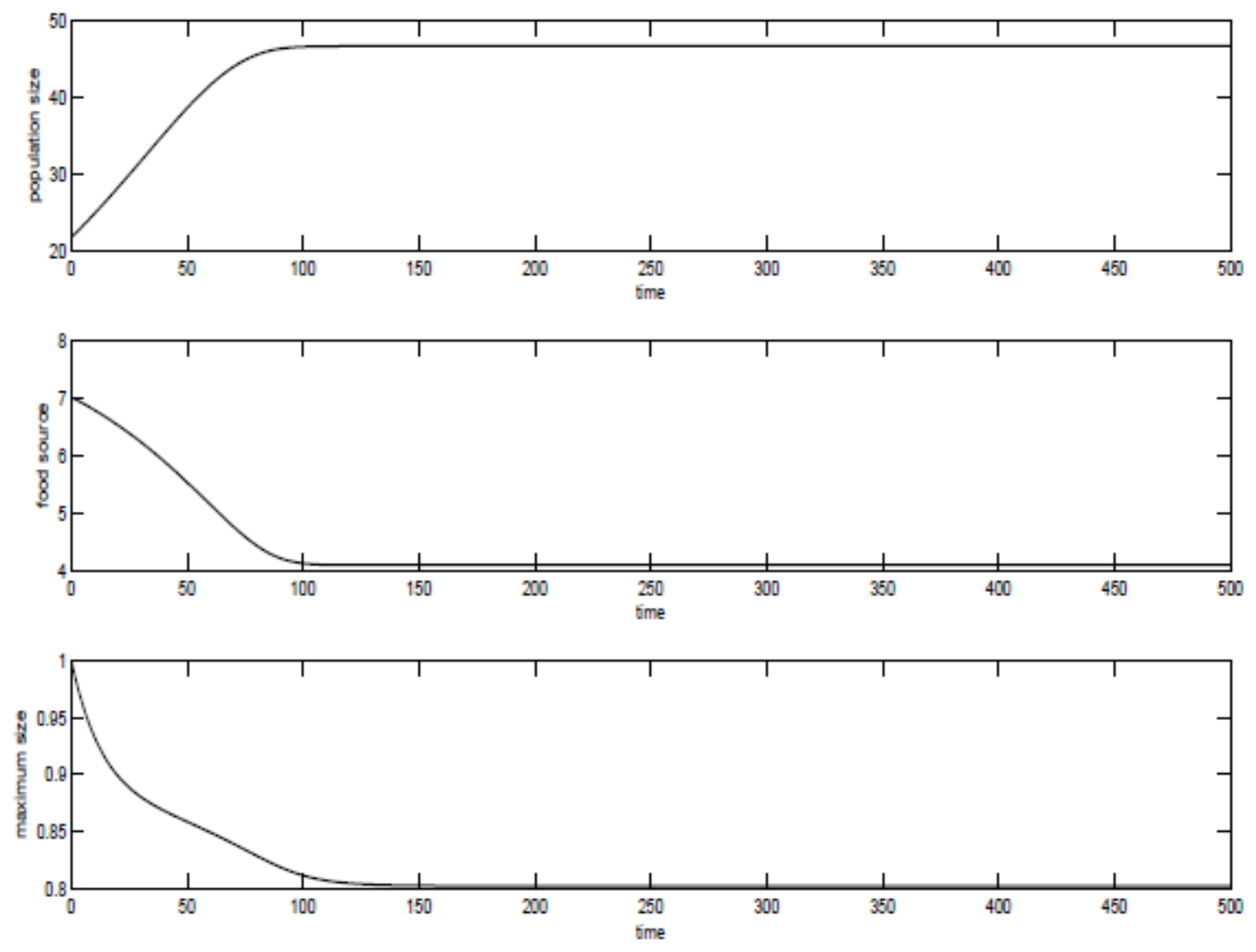

Figure 6: Evolution of the numerical solution obtained with the EM method. Stable equilibrium case, large perturbations.

Note that the numerical approximations are attracted to the equilibrium values and such an approximation remains stable in a long-time integration.

Taking into account the good behavior of the numerical scheme in a predictable situation, now we consider it in order to explore the rich dynamics of the example. As the value of the parameter $K$ in (3.4) increases, the equilibrium state becomes unstable. An example of this situation is obtained by taking $K=12$. This modification in the parameter does not have an effect on the equilibrium values $x_{M}^{*}$ and $S^{*}$ obtained in the previous experiments. But the equilibrium state (3.8) depends on $K$ and so does the total population (in this case $P^{*} \approx 60.63093240$ ). We take 
as an initial condition the equilibrium solution and the results obtained with the numerical method are presented in Figure 7.
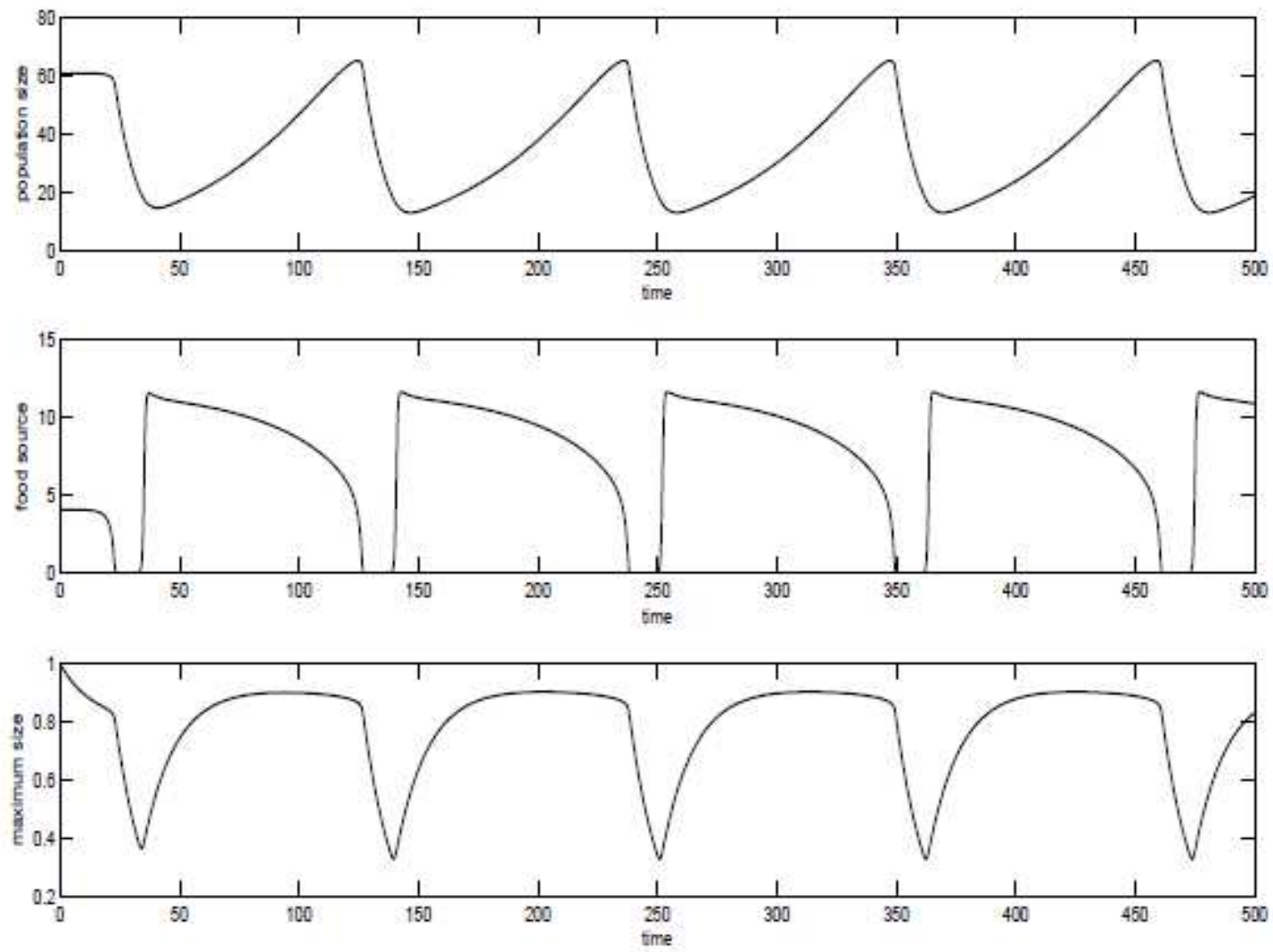

Figure 7: Evolution of the numerical solution obtained with the EM method. Unstable equilibrium case.

In this case, we can observe the instability of the equilibrium and that the solution evolves towards a cycled situation.

\section{Conclusions}

We have study the long time numerical approximation of a size-structured population model whose dependency on the environment is managed by the evolution of a vital resource. To this end, we have considered different modifications of a method employed in the solution of a fully nonlinear size-structured population model. Experimentally, the convergence of all of them is second order. We have found that the behavior of some of the methods presented is unstable. This instability is caused by two approximation problems. On one hand, the evolution of the resource is governed by a ODE so, when we are interested in the long time integration, we have to look for the discretization 
parameters belong to the absolute stability region of the numerical method. On the other hand, the evolution of the grid is also given by a system of ODEs that seems to be less sensitive to this fact. However, when we are close to the steady state, last grid nodes are close to the equilibrium and they could present stability problems. It should be noted that the approximation to the density function shows a nice behavior due to its exponential representation.

With this knowledge, we have proposed a second order numerical method that, experimentally, shows good stability properties in the approximation of the steady state. Moreover, when the steady state is unstable we have used it to analyzed the dynamics, appearing a limit cycle, and a good approximation to the bifurcation process has been obtained.

\section{Acknowledgements}

The authors are grateful to both anonymous referees for their valuable help to improve the manuscript.

The authors were supported in part by the project of the Ministerio de Educación y Ciencia MTM2008-06462-C02-02, FEDER, by the project of the Junta de Castilla y León VA046A07 and by the 2009 Grant Program for Excellence Research Group (GR137) of the Junta de Castilla y León.

\section{References}

[1] L. M. Abia, O. Angulo, J. C. López-Marcos. Age-structured population dynamics models and their numerical solutions. Ecol. Model., 188 (2005), 112-136.

[2] L. M. Abia, O. Angulo, J. C. López-Marcos. Size-structured population dynamics models and their numerical solutions. Discrete Contin. Dyn. Syst. B, 4 (2004), 1203-1222.

[3] L.M. Abia, O. Angulo, J.C. López-Marcos, M.A. López-Marcos. Numerical schemes for a size-structured cell population model with equal fission. Mat. Computer Model., 50 (2009), 653-664.

[4] M. Adimy, O. Angulo, F. Crauste, J.C. López-Marcos. Numerical integration of a mathematical model of hematopoietic stem cell dynamics. Computers and Math. Applic., 56 (2008), 594-606.

[5] O. Angulo, J. C. López-Marcos. Numerical integration of fully nonlinear size-structured models. Appl. Numer. Math., 50 (2004), 291-327.

[6] M.A. Bees, O. Angulo, J.C. López-Marcos, D. Schley. Dynamics of a structured slug population model in the absence of seasonal variation. Math. Mod. Meth. in Appl. Sci., 16 (2006), 1961-1985. 
[7] J.M. Cushing. An Introduction to Structured Populations Dynamics. CMB-NSF Regional Conference Series in Applied Mathematics. SIAM, 1998.

[8] M. Iannelli. Mathematical Theory of Age-Structured Population Dynamics. Applied Mathematics Monographs. C.N.R., Giardini Editori e Stampatori, Pisa, 1995.

[9] M. Iannelli, T. Kostova, F.A. Milner. A fourth-order method for numerical integration of age- and size-structured population models. Numer. Methods Partial Differential Equations, 25 (2009) 918-930.

[10] S.A.L.M Kooijman, J.A.J. Metz. On the dynamics of chemically stressed populations: the deduction of a population consequences from effects on individuals. Ecotox. Environ. Saf., 8 (1984), 254-274.

[11] J.A.J. Metz and E.O. Dieckmann, editors. The Dynamics of Physiologically Structured Populations. Springer Lecture Notes in Biomathematics, 68. Springer, Heildelberg, 1986.

[12] B. Perthame. Transport Equations in Biology. Birkhäuser Verlag, Basel, 2007.

[13] A.M. de Roos. Numerical methods for structured population models: The escalator boxcar train. Numer. Methods Partial Differential Equations, 4 (1988), 173-195.

[14] J. Shen, C.W. Shu, M.P. Zhang. A high order WENO scheme for a hierarchical size-structured population model. J. Sci. Comput., 33 (2007), 279-291.

[15] G.F. Webb. Theory of Nonlinear Age-Dependent Population Dynamics. Marcel Dekker, eds, New York, 1985. 